\title{
Mammakarzinom: modifizierter Prognosescore bei Hirnmetastasen
}

\author{
Verschiedene Indizes stehen für die \\ Abschätzung des Gesamtüberlebens \\ von Patientinnen mit zerebral \\ metastasiertem Brustkrebs zur \\ Verfügung. In einem erweiterten \\ Score wird auch die Anzahl der \\ Metastasen berücksichtigt.
}

D as Risiko von Hirnmetastasen bei $\mathrm{Pa}$ tientinnen mit einem Mammakarzinom steigt von 3-6\% in Frühstadien auf bis zu 30\% im Erkrankungsstadium IV. Als wichtige Entscheidungshilfen für die Auswahl der individuell erfolgversprechendsten Therapiestrategie dienen Prognosescores wie der brustkrebsspezifische Breast-GPA (Graded Prognosis Assessment). Dieser umfasst als unabhängige Prognosefaktoren den Tumorsubtyp entsprechend HER2-, Östrogen- und Progesteron-Rezeptorstatus, Karnofsky-Performance-Status (KPS) und Lebensalter. Andere prognostische Modelle berücksichtigten auch die Faktoren Lymphopenie und Hormonrezeptor-Negativität. Die Anzahl der Hirnmetastasen fand in keines dieser Modelle Eingang, obwohl sie in hohem Maß das therapeutische Vorgehen bestimmt. Die Forscher entwickelten einen modifizierten, um den Faktor „Anzahl der zerebralen Metastasen“ erweiterten Breast-GPA und verglichen dessen prognostische Aussagekraft mit der des ursprünglichen Breast-GPA mithilfe des KonkordanzIndex. Sie stützten sich dabei auf retrospektiv recherchierte Daten von 1.552 Patientinnen, die zwischen 1996 und 2013 mit zerebralen Metastasen erstdiagnostiziert wurden. Sowohl der drei Faktoren umfassende Breast-GPA als auch die Anzahl der Hirnmetastasen (weniger als 3 gegenüber 3 und mehr) erwiesen sich als unabhängige Prognosefaktoren für das Gesamtüberleben. Die Konkordanzindizes für den Breast-GPA- und modifizierten Breast-GPA-Score betrugen 0,77 bzw. 0,84 .

Fazit: Der modifizierte Breast-GPA umfasst 4 einfache klinische Parameter mit hoher prognostischer Signifikanz. Dieser Index kann einen wichtigen Beitrag leisten in der Abschätzung von Prognose und Therapieentscheidung für die individuelle Patientin.

Wolfgang Zimmermann

Subbiah IM et al. Validation and development of a modified breast graded prognostic assessment as a tool for survival in patients with breast cancer and brain metastases. J Clin Oncol. 2015;33(20):2239-45.

\section{Lymphknotenbestrahlung bei Brustkrebs nicht lebensverlängernd}

Bei nodalpositivem oder Hochrisiko-nodalnegativem Brustkrebs verringert eine Radiatio der regionalen Lymphknoten zusätzlich zur Ganzbrustbestrahlung zwar die Rezidivrate, verlängert aber nicht das Gesamtüberleben.

$\mathrm{N}$ ach einer brusterhaltenden Operation wird bei vielen Brustkrebspatientinnen die gesamte Brust bestrahlt, um die Rate der Lokalrezidive zu senken. Kanadische Forscher untersuchten, ob eine regionale Lymphknotenbestrahlung (RNI, „regional nodal irradiation“) zusätzlich zur Ganzbrustbestrahlung (WBI, „whole-breast irradiation“) die Ergebnisse verbessert. Zwischen 2000 und 2007 nahmen 1.832 Frauen an der Studie NCIC-CTG MA.20 teil. Die Patientinnen litten an nodalpositivem oder Hochrisiko-nodalnegativem Brustkrebs und hatten zuvor eine brusterhaltende Operation und eine adjuvante systemische Therapie erhalten. Sie bekamen 2:1 randomisiert entweder eine WBI plus RNI oder nur eine WBI (Kontrollgruppe). Primärer Endpunkt war das Gesamtüberleben (OS). Als sekundäre Endpunkte wurden das krankheitsfreie Überleben (DFS), das DFS bis zum Auf- treten isolierter lokoregionärer Rezidive sowie das DFS bis zum Auftreten ferner Rezidive festgelegt.

Das mediane Follow-up betrug 9,5 Jahre. Hinsichtlich der Rate für das 10-Jahres-OS war kein signifikanter Unterschied zwischen den Gruppen erkennbar: 82,8 \% in der RNI-Gruppe und $81,8 \%$ in der Kontrollgruppe (Hazard Ratio [HR] 0,91; $95 \%$-Konfidenzintervall [95\%-KI] $0,72-1,13 ; \mathrm{p}=0,38)$. Jedoch zeigte sich in einer Subgruppenanalyse, dass Patientinnen mit Östrogenrezeptor(ER)-negativem Brustkrebs in der RNI-Gruppe eine signifikant höhere 10-Jahres-OS-Rate hatten als in der Kontrollgruppe (81,3 vs. 73,9\%; HR 0,69; $95 \%$-KI 0,47-1,00; $\mathrm{p}=$ $0,05)$

Die Rate für das 10-Jahres-DFS lag in der RNI-Gruppe bei 82,0\% im Vergleich $\mathrm{zu} 77,0 \%$ in der Kontrollgruppe (HR 0,76; $95 \%$-KI 0,61-0,94; $\mathrm{p}=0,01)$. Die
Raten für das krankheitsfreie 10-JahresÜberleben bis zum Auftreten isolierter lokoregionärer Rezidive waren $95,2 \%$ bei der RNI-Gruppe versus $92,2 \%$ bei der Kontrollgruppe (HR 0,59; $95 \%$-KI 0,39$0,88 ; \mathrm{p}=0,009)$ und bis zum Auftreten ferner Rezidive 86,3 versus $82,4 \%$ (HR 0,76; 95\%-KI 0,60-0,97; $\mathrm{p}=0,03$ ).

Patientinnen in der RNI-Gruppe litten im Vergleich zur Kontrollgruppe häufiger unter akuter Pneumonitis (1,2 vs. $0,2 \% ; \mathrm{p}=0,01)$ und Lymphödemen $(8,4$ vs. $4,5 \% ; \mathrm{p}=0,001)$ von $\mathrm{Grad} 2$ oder stärker. Weitere Nebenwirkungen waren z. B. Strahlendermatitis als akute sowie Teleangiektasie als verzögerte Effekte.

Fazit: Frauen mit nodalpositivem Brustkrebs oder Hochrisiko-nodalnegativem Brustkrebs, die zusätzlich zur WBI eine RNI erhalten, überleben zwar nicht länger, profitieren aber von einer geringeren Rezidivrate.

Christine Cramer

Whelan TJ et al. Regional Nodal Irradiation in Early-Stage Breast Cancer. N Engl J Med. 2015;373(4):307-16. 